\title{
Spread of Carbapenemase-producing Enterobacteria in a Southwest Hospital in China
}

\author{
Sheng Chen², Wei Feng ${ }^{1}$, Jianhong Chen ${ }^{1}$, Wei Liao², Nianhai $\mathrm{He}^{2}$, Qian Wang ${ }^{3}$, Fengjun Sun ${ }^{\text {* }}$ and Peiyuan Xia ${ }^{\text {* }^{*}}$
}

\begin{abstract}
Background: The rapid emergence and dissemination of carbapenem resistance in Enterobacteriaceae complicates the treatment of infections caused by these organisms.

Methods: We collected clinical isolates with meropenem inhibition zones of $\leq 22 \mathrm{~mm}$ from January 1, 2009, through December 31, 2010. We attempted to amplify the NDM-1 gene from these isolates and conducted the modified Hodge test (MHT). The minimal inhibitory concentration (MIC) of the MHT-positive strains was determined by the agar disk dilution method. The carbapenemase-encoding resistance genes of these strains were examined using polymerase chain reaction (PCR) analysis and a sequencing strategy to characterize these enzymes. The clonal relationship among isolates was analyzed by pulsed-field gel electrophoresis (PFGE).
\end{abstract}

Results: Among the 158 Enterobacteriaceae isolates that were collected, there were no NDM-1-positive strains and 26 MHT-positive strains. Among the latter, 18 strains were IMP-4-positive, and 1 was KPC-2-positive. In addition, 15 of the IMP-4-positive Klebsiella pneumoniae strains belonged to 4 PFGE genotypes, with 8 strains having the same genotype.

Conclusion: These results suggest that nosocomial infections are one of the main reasons for the spread of these resistant strains.

Keywords: Enterobacteria, Carbapenemase, Emergence, Spread, Resistance

\section{Introduction}

Enterobacteriaceae are the most common pathogenic bacteria. Recently, the emergence of resistant pathogenic bacteria has caused significant problems for the clinical treatment of enterobacterial infections worldwide. There are many mechanisms leading to this resistance [1]. Extendedspectrum $\beta$-lactamase (ESBL)-producing pathogenic enterobacteria is a serious problem for antibiotic management because the ESBL genes are easily transferred from one organism to another via plasmids or other mobile genetic elements in combination with non- $\beta$-lactam resistance mechanisms, leading to multidrug-resistant isolates $[1,2]$.

Carbapenem antibiotics are a class of $\beta$-lactam antibiotics that are often used as a last resort for treating enterobacterial resistance. However, an increasing number of studies are reporting carbapenem-resistant enterobacteria [2-4]. Carbapenem-resistant enterobacterial infections are now clearly associated with significant morbidity and

\footnotetext{
*Correspondence: fengj_sun@163.com; peiyuan_xia@aliyun.com 'Department of Pharmacy, Southwest Hospital, Third Military Medical University, Chongqing 400038, China

Full list of author information is available at the end of the article
}

mortality. However, few studies have monitored the spread of these Enterobacteriaceae. Here, we report the spread of IMP-4-producing enterobacteria in a hospital in Chongqing, China.

\section{Materials and methods \\ Study design}

The study period was from January 1, 2009, through December 31, 2010. All samples were collected from the Southwest Hospital, which is one of the largest teaching hospitals in Chongqing, China, with approximately 3000 beds. The bacterial isolates were identified in the clinical microbiology laboratory using standard biochemical tests and the Vitek system (BioMérieux, Hazelwood, MO). If the meropenem inhibition zone was $\leq 22 \mathrm{~mm}$, an attempt was made to amplify the NDM-1 gene from the strain, and the strain was evaluated with the modified Hodge test (MHT) [5]. The MHT has been widely used to screen for carbapenemase activity because it directly analyzes this activity. If the sample was either MHTpositive or NDM-1-positive, the medical records and 
laboratory data for the associated patient were retrospectively reviewed using records available from the Southwest Hospital information system and from the microbiology laboratory. In this study, each isolate was collected from a different patient. This study was approved by the Ethics Committee of the Third Military Medical University (Approval number, KY200508).

\section{Susceptibility testing}

Bacterial isolates were identified according to standard methods and tested against various antibiotics by the disk diffusion method; the zone diameters were interpreted according to the Clinical and Laboratory Standards Institute (CLSI) guidelines. The minimal inhibitory concentration (MIC) was evaluated for MHT-positive and NDM-1positive strains. The MIC was determined by the agar disk dilution method in freshly prepared test medium (MH) according to the 2010 guidelines of the CLSI, and Escherichia coli strain ATCC 25922 was used as the control strain for the MIC testing. The resistance rate refers to the number of resistant strains divided by the total number of strains. The susceptible strains include those that are fully susceptible and those with an intermediate susceptible according to the CLSI standard.

\section{PFGE}

All Klebsiella pneumoniae isolates were analyzed by pulsed-field gel electrophoresis (PFGE) using the contourclamped homogeneous electric field (CHEF) technique, which is similar to the method described by Shen et al. [6] and Gouby et al. [7]. The XbaI restriction enzyme was used (Takara, DaLian, China). DNA fragments were separated by electrophoresis in a $1 \%$ agarose III gel (Bio-Rad, China) with a CHEF apparatus (CHEF Mapper XA, Bio$\mathrm{Rad})$. The electrophoresis was performed at $14^{\circ} \mathrm{C}$ and $6 \mathrm{~V} /$ $\mathrm{cm}$ and with alternating pulses at a $120^{\circ}$ angle in a 2 - to 40-s pulse-time gradient for $24 \mathrm{~h}$. The typing criteria were based on the protocol described by Shen et al. [6] and Tenover et al. [8].

\section{Carbapenemase analysis}

Carbapenemase genes that are common in China were amplified by PCR with the primers shown in Table 1. NDM-1-, IMI-1-, SPM-1-, IMP-1-, KPC-1-, VIM-1-, and OXA-typepositive strains were preserved in our laboratory [9]. The sequence analyses were performed using the BLAST program available on the National Center for Biotechnology Information server (http://www.ncbi.nlm.nih.gov/).

\section{Results}

From January 1, 2009 to December 31, 2010, 158 Enterobacteriaceae isolates (92 K. pneumoniae, $9 \mathrm{E}$. coli, and 57 Enterobacter cloacae) with reduced susceptibility to imipenem were identified in the hospital. Only 1 isolate of each
Table 1 The primers used to amplify the carbapenemase genes by PCR

\begin{tabular}{|c|c|c|c|}
\hline Gene & Primer & Size (bp) & Reference \\
\hline IMP-1 F & 5'-atgagcaagttatctgtattctttat-3' & 741 & 9 \\
\hline IMP-1R & $5^{\prime}$-ttagttgcttagttttgatggttt-3' & & \\
\hline KPC-1 F & $5^{\prime}$-tcgccgtctagttctgctgtctt-3' & 965 & 9 \\
\hline KPC-1R & $5^{\prime}$-ccgcgcagactcctagcctaa-3' & & \\
\hline NDM-1 F & 5'-tcaccgagattgccgagcga-3' & 457 & 9 \\
\hline NDM-1R & 5'-gggcagtcgcttccaacggt-3' & & \\
\hline VIM-1 F & 5'- ggtcgcatatcgcaacgcagt-3' & 636 & 9 \\
\hline VIM-1R & $5^{\prime}$-cggcgactgagcgatttttg-3' & & \\
\hline IMI-1 F & 5'- ccattcacccatcacaac-3' & 440 & 9 \\
\hline IMI-1R & 5'- ctaccgcataatcatttgc-3' & & \\
\hline SPM-1 F & $5^{\prime}-$ ctgcttggattcatgggcgc-3' & 783 & 1 \\
\hline SPM-1R & $5^{\prime}-$ ccttttccgcgaccttgatc- $3^{\prime}$ & & \\
\hline OXA-23 F & $5^{\prime}-$ acttgctatgtggttgcttctctt-3' & 797 & 9 \\
\hline OXA-23R & 5'- ttcagctgttttaatgatttcatca-3' & & \\
\hline OXA-24 F & $5^{\prime}$ - cgatcagaatgttcaagcgc-3' & 559 & 9 \\
\hline OXA-24R & $5^{\prime}-$ acgattctcccctctgcgc-3' & & \\
\hline OXA-48 F & $5^{\prime}-$ ttggtggcatcgattatcgg-3' & 744 & 1 \\
\hline OXA-48R & $5^{\prime}$ - gagcacttcttttgtgatggc-3' & & \\
\hline OXA-51 F & 5'- tccaaatcacagcgcttcaaaa-3' & 639 & 9 \\
\hline OXA-51R & $5^{\prime}$ - tgaggctgaacaacccatcca-3' & & \\
\hline OXA-58 F & $5^{\prime}$ - cgatcagaatgttcaagcgc-3' & 529 & 1 \\
\hline OXA-58R & $5^{\prime}-$ acgattctcccctctgcgc- $3^{\prime}$ & & \\
\hline
\end{tabular}

bacterial species was selected per patient, and all of the strains were collected from patients $48 \mathrm{~h}$ after admission. Among the 158 Enterobacteriaceae, the Hodge test was positive for 26 strains ( $20 \mathrm{~K}$. pneumoniae, $4 \mathrm{E}$. coli, and 2 E. cloacae), and no strain was NDM-1-positive. The features of these isolates and the patients are presented in Table 2. Among twenty-six clinical strains, eighteen isolates were IMP-4 positive, and one was KPC-2 positive. Seventeen strains were from sputum, and sixteen strains were from pediatrics. All the strains of group A PFGE genotype were from pediatrics.

The results of MIC range, $\mathrm{MIC}_{50}$ and $\mathrm{MIC}_{90}$ analyses are presented in Table 3 . We used previously described PCR reactions to screen for carbapenem-hydrolyzing $\beta$ lactamase genes. The PCR reactions for the IMP-4 and KPC-2 genes were positive [9]. The major epidemic pattern A consisted of ten IMP-4-positive isolates from the Department of Pediatrics. However, the only one KPC-2positive strain was collected from the Department of Neurology.

XbaI pulsed-field gel electrophoresis was then used to study the genetic relatedness of the $20 \mathrm{~K}$. pneumoniae isolates. The results are presented in Figure 1, and they suggest nosocomial transmission. 
Table 2 Features of the Enterobacteriaceae clinical isolates

\begin{tabular}{|c|c|c|c|c|c|c|c|c|c|c|c|c|}
\hline & $\begin{array}{l}\text { Collection } \\
\text { date }\end{array}$ & Strain & Gender & Age & Specimen & $\begin{array}{l}\text { Hospital } \\
\text { days }\end{array}$ & Ward & Diagnosis & $\begin{array}{c}\text { PFGE } \\
\text { genotype }\end{array}$ & $\begin{array}{l}\text { Carbapenemase } \\
\text { gene }\end{array}$ & IMP-MIC & MEM-MIC \\
\hline 1 & $2009-9-28$ & Kpn & Male & 31 days & Sputum & 11 & Pediatrics & Acute pneumonia & A & IMP-4 & 2 & 1 \\
\hline 2 & 2009-10-7 & Kpn & Female & 2 days & Blood & 7 & Pediatrics & Neonatal pneumonia & A & IMP-4 & 4 & 2 \\
\hline 3 & 2009-12-19 & Kpn & Female & 10 days & Sputum & 6 & Pediatrics & Neonatal pneumonia & A & IMP-4 & $<1$ & $<1$ \\
\hline 4 & $2009-12-26$ & Kpn & Male & 56 years & Abdominal fluid & 31 & Hepatobiliary department & Abdominal infection & C & - & 8 & 8 \\
\hline 5 & $2010-4-3$ & Kpn & Male & 30 days & Sputum & 8 & Pediatrics & Acute pneumonia & E & IMP-4 & 64 & 128 \\
\hline 6 & 2010-4-10 & Eco & Female & 57 years & Abdominal fluid & 21 & ICU & Severe acute pancreatitis & - & IMP-4 & 4 & 2 \\
\hline 7 & 2010-5-15 & $\mathrm{Ecl}$ & Male & 15 days & Sputum & 22 & Pediatrics & Neonatal pneumonia & - & IMP-4 & 4 & 8 \\
\hline 8 & $2010-5-24$ & Kpn & Male & 58 minutes & Sputum & 11 & Pediatrics & Neonatal pneumonia & A & IMP-4 & 4 & 4 \\
\hline 9 & $2010-5-26$ & Kpn & Male & 38 minutes & Sputum & 11 & Pediatrics & Neonatal pneumonia & E & IMP-4 & 16 & 16 \\
\hline 10 & $2010-5-29$ & Kpn & Male & 2 months & Sputum & 21 & Pediatrics & Acute pneumonia & B & IMP-4 & 128 & 64 \\
\hline 11 & 2010-5-29 & Kpn & Male & 6 hours & Sputum & 12 & Pediatrics & Neonatal pneumonia & A & IMP-4 & 4 & 4 \\
\hline 12 & $2010-6-3$ & Kpn & Female & 50 minutes & Sputum & 88 & Pediatrics & Neonatal pneumonia & A & - & 4 & 2 \\
\hline 13 & $2010-6-29$ & Kpn & Female & 19 days & Sputum & 12 & Pediatrics & Neonatal pneumonia & A & IMP-4 & $<1$ & $<1$ \\
\hline 14 & 2010-6-29 & Eco & Male & 56 years & Ascites & 37 & Department of hepatobiliary & Carcinoma of the pancreatic head & - & IMP-4 & $<1$ & $<1$ \\
\hline 15 & 2010-7-3 & Kpn & Male & 20 years & Sputum & 49 & ICU & Open craniocerebral injury & E & - & 4 & 4 \\
\hline 16 & 2010-7-3 & Eco & Male & 79 years & Sputum & 6 & Department of gastroenterology & Chordapsus, pneumonia & - & - & $<1$ & $<1$ \\
\hline 17 & 2010-7-17 & Kpn & Male & 1 hour & Sputum & 9 & Pediatrics & Neonatal pneumonia & A & IMP-4 & 1 & $<1$ \\
\hline 18 & $2010-7-21$ & Kpn & Male & 1 hour & Sputum & 7 & Pediatrics & Neonatal pneumonia & A & - & 2 & 1 \\
\hline 19 & $2010-7-21$ & Eco & Female & 44 years & Blood & 46 & Department of hepatobiliary & Congenital choledochus cyst & - & - & 2 & 1 \\
\hline 20 & $2010-7-23$ & Kpn & Female & 16 days & Sputum & 10 & Pediatrics & Neonatal pneumonia & E & IMP-4 & 32 & 16 \\
\hline 21 & $2010-7-23$ & Kpn & Male & 1 hour & Sputum & 18 & Pediatrics & Neonatal pneumonia & C & IMP-4 & 32 & 32 \\
\hline 22 & $2010-7-23$ & Kpn & Male & 60 years & Blood & 46 & ICU & Multiple injuries & E & IMP-4 & 8 & 8 \\
\hline 23 & 2010-10-11 & Kpn & Female & 26 days & Sputum & 12 & Pediatrics & Neonatal pneumonia & A & IMP-4 & 2 & 2 \\
\hline 24 & 2010-10-17 & $\mathrm{Ecl}$ & Male & 54 years & Wound & 48 & $\mathrm{ICU}$ & Penetrating wound & - & - & 1 & $<1$ \\
\hline 25 & 2010-10-20 & Kpn & Female & 69 years & Wound & 17 & Department of neurology & Pneumonia & D & KPC-2 & 16 & 8 \\
\hline 26 & 2010-10-20 & Kpn & Female & 64 years & Wound & 34 & Burn & Burn & B & IMP-4 & 2 & 2 \\
\hline
\end{tabular}


Table 3 MIC range, $\mathrm{MIC}_{50}$ and $\mathrm{MIC}_{90}$ for antibiotics tested against Enterobacteriaceae

\begin{tabular}{llll}
\hline Antimicrobial agent & MIC range $(\boldsymbol{\mu} \mathbf{g} / \mathbf{m l})$ & MIC $_{\mathbf{5 0}}(\boldsymbol{\mu g} / \mathbf{m l})$ & $\mathbf{M I C}_{\mathbf{9 0}}(\boldsymbol{\mu \mathbf { g } / \mathbf { m l } )}$ \\
\hline Imipenem & $1<-128$ & 4 & 16 \\
Meropenem & $1<-64$ & 2 & 16 \\
Ceftazidim & $1<->512$ & 512 & $>512$ \\
Ceftriaxone & $1<->512$ & $>512$ & $>512$ \\
Piperacillin/tazobactam & $1<->512$ & 128 & 512 \\
Piperacillin & $1->512$ & $>512$ & $>512$ \\
Ciprofloxacin & $1<-64$ & 4 & 32 \\
Levofloxacin & $1<-128$ & 8 & 32 \\
Gentamicin & $1<->512$ & 8 & 64 \\
Tobramycin & $1<->512$ & 4 & 64 \\
\hline
\end{tabular}

\section{Discussion}

In the present study, we analyzed the carbapenemase genes and assessed the spread of IMP-4-positive enterobacteria in a hospital in Chongqing, China. IMP-4 is a common MBL and was first reported in Acinetobacter baumannii in Hong Kong, China; it subsequently spread throughout the world, particularly to mainland China and Australia [10,11]. IMP-
4 has been reported in enterobacteria in China [12] but has not been found in Southwestern China. Previously, we found that the IMP-4 gene existed in A. baumannii (data were not shown) and Pseudomonas aeruginosa [9]. Since 2007, we have detected the carbapenemase genes in enterobacteria. In 2009, we found the first carbapenemase gene, IMP-4, which we subsequently showed to be spreading

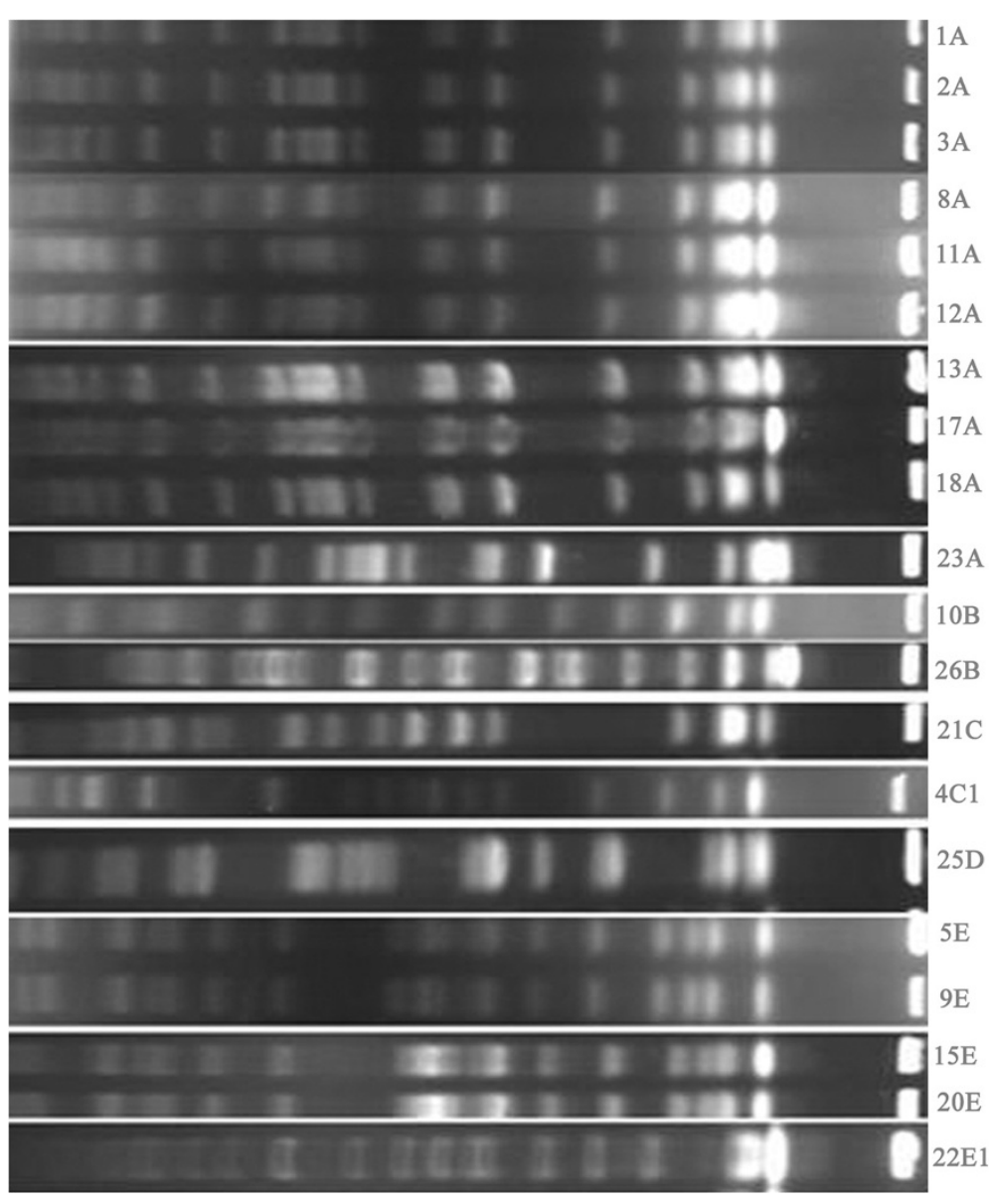

Figure 1 PFGE analysis of genomic DNA from isolates of $K$. pneumoniae. 
among enterobacteria. However, IMP-8 has been reported in Chongqing despite the presence of IMP-4 [13]. These results suggested that IMP-4 may be transferred to enterobacteria from other bacteria in our hospital. After the first IMP-4-positive $K$. pneumoniae strain was found in the Pediatrics Unit, 18 IMP-4-positive enterobacteria were identified, including one E. cloacae and two E. coli strains. KPC-2 is common in the coastal cities of China, such as Hangzhou and Shanghai $[14,15]$. Only one KPC-2-positive strain was found among the clinical isolates, and it was reported in The First Affiliated Hospital of Chongqing Medical University, Chongqing [13]. Since then, we have not found another KPC-2-positive strain. Thus, the KPC-2positive strain may have been present in the patient prior to hospitalization and not acquired from a hospital ward.

For seven MHT-positive strains, no carbapenemase gene was amplified. The MHT has negative and positive likelihood rates, and the positive likelihood rate in our experiment is higher than that in a previous report [16]. Here, we only tested for the commonly appearing carbapenemase genes in China, so it is possible that these seven strains have other resistance genes. The MHTand IMP-4-positive strains exhibited low-level resistance and even susceptibility to carbapenems in our study, and Nordmann reported similar results [2]. Thus, the highly resistant strains carrying IMP-4 must have additional mechanisms for carbapenem resistance, such as outermembrane permeability defects, other resistance genes, or target alteration. Therefore, to treat carbapenemasepositive strains, carbapenem antibiotics, although not the preferred choice, may be the only choice.

The PFGE revealed genetic diversity among the $20 \mathrm{~K}$. pneumoniae isolates, which belonged to five PFGE types. Ten strains of K. pneumoniae shared the same PFGE type. The phenomenon of the same clones spreading within the same wards or throughout different wards during the same period was observed. The same clones had similar resistance results but slightly different MIC results, suggesting that some features of the bacteria had changed as they spread and replicated or that these bacteria could easily exchange mobile genetic elements. These findings indicated that the decreased susceptibility to carbapenems in K. pneumoniae may arise by the stepwise accumulation of multiple carbapenem resistance determinants in different clones. These results suggest that this type of infection can be classified as a hospital-derived infection. Therefore, controlling hospital infections is a method for reducing the accumulation of bacteria resistance determinants.

The average hospital stay of the patients with these isolates was 23.1 days, while the average hospital stay of all patients in our hospital was 9.8 days. Long-term acute care hospitals (LTACHs) may play a particularly important role in the spread of KPC-producing Enterobacteriaceae, particularly among neonates or patients with a serious illness who have a reduced immune system functional capacity and who are undergoing invasive examinations or treatments. Our results support the conclusions reported by Endimiani [17]. Thus, for patients with a high risk of infection, reinforcement of infection control measures based on the guidance for carbapenemase-producing Enterobacteriaceae prevention [17] with early warning is particularly critical and should include promotion of hand hygiene. In addition, the immediate initiation of contact precautions is necessary, which can lead to a considerable reduction in hospital-acquired carbapenemase producers. Our findings also suggest that a significant containment of carbapenem-resistant $K$. pneumoniae strains from the environment is possible with the implementation of a comprehensive infection control intervention program that includes active surveillance, carrier isolation/cohorting, and a dedicated staff to treat individuals who become infected. The advent of these tests has enabled the early detection of clonal strains that are likely to be introduced into our hospital, especially from patients with previous hospitalizations. In the future, we will analyze more diverse strains and resistant genes. After infection control measures are reinforced, we will conduct additional infection control studies to test the effects of reinforcing infection control measures.

Taken together, these findings reveal the process involved in the recent spread of IMP-4 in our hospital. Carbapenem-resistant enterobacteria have become a significant concern in our hospital. The presence of carbapenemases, such as KPC-2 and IMP-4, may have played a significant role in the development of these resistant strains. Cooperation among the clinical microbiology laboratory, the infection control team, and the medical and nursing staff is vital for the design and implementation of appropriate infection control measures for carbapenemase-producing pathogens. The use of active surveillance as part of this multifactorial intervention may also aid in decreasing the secondary transmission rates of imported genes. Other interventions, such as shortening the average length of hospital stays, may further augment these infection control actions. Examining whether our interventions change the prevalence of other, less common, multidrug-resistant pathogens will also be of interest.

\section{Competing interests}

The authors declare that they have no competing interests.

\section{Authors' contributions}

SC carried out the protocol design and drafted this manuscript. WF carried out the collection and analysis of data for this study, and revised the content. JC carried out the collection of data, the analysis and interpretation of these data. WL, NH and QW participated in the collection of data for this study. FS and PX carried out the protocol design, collection of data, the analysis and interpretation of these data, and drafted and revised the content of this manuscript. All authors read and approved the final manuscript. 


\section{Author details}

${ }^{1}$ Department of Pharmacy, Southwest Hospital, Third Military Medical University, Chongqing 400038, China. ²Department of Pediatrics, Southwest Hospital, Third Military Medical University, Chongqing, China. ${ }^{3}$ Department of

Pharmacy, Xinqiao Hospital, Third Military Medical University, Chongqing, China.

Received: 13 June 2014 Accepted: 3 August 2014

Published: 12 August 2014

\section{References}

1. Logan LK: Carbapenem-resistant enterobacteriaceae: an emerging problem in children. Clin Infect Dis 2012, 55:852-859.

2. Nordmann P, Naas T, Poirel L: Global spread of carbapenemase-producing Enterobacteriaceae. Emerg Infect Dis 2011, 17:1791-1798.

3. Voulgari E, Poulou A, Koumaki V, Tsakris A: Carbapenemase-producing Enterobacteriaceae: now that the storm is finally here, how will timely detection help us fight back? Future Microbiol 2013, 8:27-39.

4. Thurlow CJ, Prabaker K, Lin MY, Lolans K, Weinstein RA, Hayden MK, Centers for Disease Control and Prevention Epicenters Program: Anatomic sites of patient colonization and environmental contamination with klebsiella pneumoniae carbapenemase-producing Enterobacteriaceae at long-term acute care hospitals. Infect Control Hosp Epidemiol 2013, 34:56-61.

5. Lee K, Chong Y, Shin HB, Kim YA, Yong D, Yum JH: Modified Hodge and EDTA-disk synergy tests to screen metallo- $\beta$-lactamase-producing strains of pseudomonas and acinetobacter species. Clin Microbiol Infect 2001, 7:88-91.

6. Shen P, Wei Z, Jiang Y, Du X, Ji S, Yu Y, Li L: Novel genetic environment of the carbapenem-hydrolyzing $\beta$-lactamase KPC-2 among Enterobacteriaceae in China. Antimicrob Agents Chemother 2009, 53:4333-4338.

7. Gouby A, Neuwirth C, Bourg G, Bouziges N, Carles-Nurit MJ, Despaux E, Ramuz M: Epidemiological study by pulsed-field gel electrophoresis of an outbreak of extended-spectrum $\beta$-lactamase-producing Klebsiella pneumoniae in a geriatric hospital. J Clin Microbiol 1994, 32:301-305.

8. Tenover FC, Arbeit RD, Goering RV, Mickelsen PA, Murray BE, Persing DH, Swaminathan B: Interpreting chromosomal DNA restriction patterns produced by pulsed-field gel electrophoresis: criteria for bacterial strain typing. J Clin Microbiol 1995, 33:2233-2239.

9. Sun FJ, Shi HQ, Zhang XB, Fang YD, Chen YC, Chen JH, Wang Q, Yang B, Feng W, Xia PY: Detection of carbapenemase-encoding genes among clinical isolates of Pseudomonas aeruginosa in a burn unit of China. J Burn Care Res 2013, 34:453-458.

10. Zhao $\mathrm{WH}, \mathrm{Hu} Z \mathrm{ZQ}$ : IMP-type metallo- $\beta$-lactamases in gram-negative bacilli: distribution, phylogeny, and association with integrons. Crit Rev Microbiol 2011, 37:214-226.

11. Chu YW, Afzal-Shah M, Houang ET, Palepou MI, Lyon DJ, Woodford N, Livermore DM: IMP-4, a novel metallo- $\beta$-lactamase from nosocomial Acinetobacter spp. collected in Hong Kong between 1994 and 1998. Antimicrob Agents Chemother 2001, 45:710-714.

12. Wei Z, Yu T, Qi Y, Ji S, Shen P, Yu Y, Chen Y: Coexistence of plasmid-mediated KPC-2 and IMP-4 carbapenemases in isolates of Klebsiella pneumoniae from China. J Antimicrob Chemother 2011, 66:2670-2671.

13. Dai W, Sun S, Yang P, Huang S, Zhang X, Zhang L: Characterization of carbapenemases, extended spectrum $\beta$-lactamases and molecular epidemiology of carbapenem-non-susceptible Enterobacter cloacae in a Chinese hospital in Chongqing. Infect Genet Evol 2013, 14:1-7.

14. Hu F, Chen S, Xu X, Guo Y, Liu Y, Zhu D, Zhang Y: Emergence of carbapenem-resistant clinical Enterobacteriaceae isolates from a teaching hospital in Shanghai, China. J Med Microbiol 2012, 61:132-136.

15. Wei ZQ, Du XX, Yu YS, Shen P, Chen YG, Li LJ: Plasmid-mediated KPC-2 in a Klebsiella pneumoniae isolate from China. Antimicrob Agents Chemother 2007, 51:763-765.
16. Mathers AJ, Carroll J, Sifri CD, Hazen KC: Modified Hodge test versus indirect carbapenemase test: prospective evaluation of a phenotypic assay for detection of klebsiella pneumoniae carbapenemase (KPC) in Enterobacteriaceae. J Clin Microbiol 2013, 51:1291-1293.

17. Centers for Disease Control and Prevention: Guidance for control of infections with carbapenem-resistant or carbapenemase-producing Enterobacteriaceae in acute care facilities. MMWR Morb Mortal Wkly Rep 2009, 58:256-260.

doi:10.1186/s12941-014-0042-4

Cite this article as: Chen et al:: Spread of Carbapenemase-producing Enterobacteria in a Southwest Hospital in China. Annals of Clinical Microbiology and Antimicrobials 2014 13:42

\section{Submit your next manuscript to BioMed Central and take full advantage of:}

- Convenient online submission

- Thorough peer review

- No space constraints or color figure charges

- Immediate publication on acceptance

- Inclusion in PubMed, CAS, Scopus and Google Scholar

- Research which is freely available for redistribution

Submit your manuscript at www.biomedcentral.com/submit
C BioMed Central 